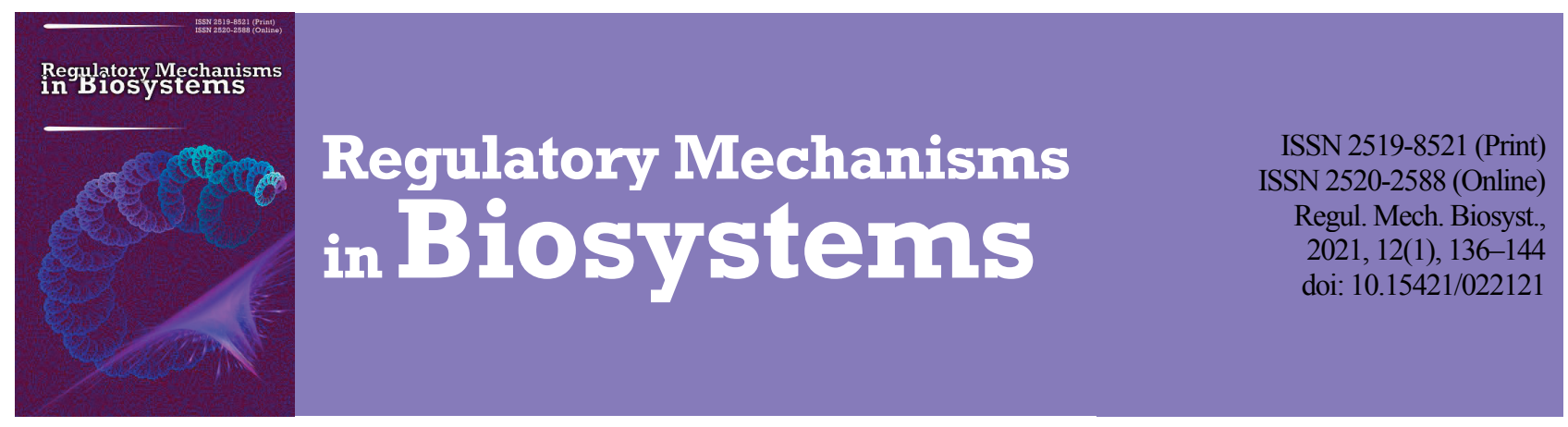

\title{
The influence of microwave electromagnetic radiation on rat heart morphogenesis during thyroidectomy
}

\author{
O. O. Drobakhin*, V. I. Magro*, V. V. Kosharnyi**, V. H. Rutgaizer**, L. V. Abdul-Ohly** \\ * Oles Honchar Dnipro National University, Dnipro, Ukraine \\ **Dnipropetrovsk Medical Academy of the Ministry of Health of Ukraine, Dnipro, Ukraine
}

Article info

Received 15.02.2021

Received in revised form 11.03.2021

Accepted 12.03.2021

Oles Honchar Dnipro National University,

Gagarin ave., 72,

Dnipro, 49010, Ukraine.

Tel.: + 38-067-589-15-95

E-mail:

drobakhino@gmail.com

Dnipropetrovsk Medical Academy of the Ministry of Health of Ukraine,

Vernadsky st., 9

Dnipro, 49044, Ukraine.

Tel.: +38-050-568-64-48.

E-mail:kosha.v@ukr.net
Drobakhin, O. O., Magro, V. I., Kosharnyi, V. V., Rutgaizer, V. H., \& Abdul-Ohly, L. V. (2021). The influence of microwave electromagnetic radiation on rat heart morphogenesis during thyroidectomy. Regulatory Mechanisms in Biosystems, 12(1), 136144. doi:10.15421/022121

It is known that electromagnetic radiation affects the functional systems of living beings, in particular, the endocrine and cardiovascular systems and directly the heart cells and their functioning. In this case, the influence of electromagnetic radiation significantly affects the properties of the cardiovascular system and changes physiological processes in it. Moreover, sensitivity to signals is higher for biosystems with a higher level of organization. The method of simulating hypothyroidism for rats makes it possible to develop an experimental model for analyzing morphogenetic changes in the rat heart. Under conditions of hypothyroidism modeling, destructive changes in muscle fibers are observed in the myocardium after thyroidectomy, but after microwave irradiation with an exposure of 45 minutes, a regenerative-compensatory reconstruction of the structural components of the myocardium of the heart wall occurs. After thyroidectomy and microwave irradiation with an exposure of 120 minutes, an increase in degenerative and destructive processes in the heart myocardium was observed. For the first time, a comparative ultramicroscopic study using electron microscopy was carried out. It showed that after electromagnetic irradiation with an exposure of 45 minutes, the general structure of the mitochondrial apparatus does not change. Irradiation with an exposure of 120 minutes causes the destruction of subsarcolemal and paranuclear organelles, edema and degradation of intermyofibrillar mitochondria. After exposure to electromagnetic radiation with the exposure of 45 minutes in a hypothyroid state, a compensatory restructuring of the energy apparatus of the contractile cardiomyocytes of the left ventricle occurs due to the formation of mitochondria and an increase in their contact interaction. After exposure to radiation for 120 minutes, the development of destructive-degenerative processes in the mitochondrial apparatus of left ventricular cardiomyocytes, deepening of damage to intermitochondrial contacts was observed. The most significant increase in the relative volume of the endothelium in the areas of the rat heart myocardium was observed for the case of microwave irradiation only (without experimental hypothyroidism) with exposure duration of 45 minutes. Immunohistochemical study both for the case of microwave irradiation only and for the case of microwave irradiation under conditions of experimental hypothyroidism allows us to conclude that morphogenetic transformations are highly active in the myocardium of the heart when the duration of exposure is 45 minutes. This study will make it possible to develop recommendations for persons with thyroid diseases on the duration of daily exposure of staying in electromagnetic field emitted by devices with characteristics similar to the case under study.

\section{Introduction}

It is known that natural and anthropogenic electromagnetic fields affect any biological object. The influence of natural electromagnetic fields is well studied. At the same time, the development of wireless communications in the context of digitalization of society leads to an increase in the overall level of anthropogenic fields (Belyaev et al., 2016; Hardell, 2017). In 2019, the International Labor Organization stated the fact of the accelerated transformation of society through the development of digital technologies. Digital technologies are widely used both in industry and in everyday life. There is a convergence of material production and the virtual world. And in such a system, electromagnetic fields are the main carriers of information. Therefore, the presence of a background electromagnetic field is an everyday reality not only in large metropolitan areas, but also in small settlements (Sagar et al., 2017; Jalilian et al., 2019; Melnick et al., 2019). Sufficiently uniform irradiation of territories is provided by satellite television systems. According to existing standards, electromagnetic radiation in the frequency range of $10.7-12.7 \mathrm{GHz}$ is used for this purpose. Any biological object in real conditions is exposed to a combination of various factors. These factors can be of different nature, for example, physical, biological, social ones. The complex influence of these factors on a living organism, especially the simultaneous effect of several of them, can lead to an increase or decrease in the resulting effects. This cumulative effect is not entirely obvious or may be absent altogether under the separate influence of such external factors.

The influence of anthropogenic electromagnetic radiation, to which a person is exposed in the process of industrial activity, has been well studied (Meroni \& Schreck, 2015). The most frequent case is the complex impact of electromagnetic radiation and factors such as noise, air temperature, and vibration on a person. The result of such complex effect can be multidirectional reactions of the living being. It is shown that the combined effect of electromagnetic radiation and low ambient temperature leads to an increase in the number of cardiovascular diseases (Miah \& Kamat, 2017). It is emphasized that high-frequency electromagnetic fields have a more negative effect on humans compared to low-frequency fields. It is indicated that a high-frequency electromagnetic field stimulates the cellular stress response, causing the breaking of DNA strands. It is noted that there is a connection between electromagnetic radiation and the develop- 
ment of malignant tumours in rats. The study also addresses the issue of the adverse effects of mobile phone exposure on children.

It is a well-proven fact that long-term exposure to low-frequency electromagnetic fields formed by near high-voltage transmission lines, sources of electromagnetic radiation and electric vehicles can have a negative effect on humans (Esmailzadeh et al., 2019; Kim et al., 2020). These papers emphasize that although such electromagnetic exposure is within normal limits, there is a need to study the long-term effects on human health. It has been found that living in close proximity to high-voltage power lines leads to a number of health problems (Esmailzadeh et al., 2019). The influence of electromagnetic fields from power lines on female fertility has been studied. Women who lived 500 meters away from a high-voltage transmission line had a higher risk of infertility than women who lived more than 1000 meters away. Various factors related to electromagnetic exposure in the course of professional activity have been investigated (Kim et al., 2020). It is shown that the results of the average annual electromagnetic exposure depend on the lifestyle, body mass index, smoking, and the length of the working day. There is a need to improve the procedures of influence of electromagnetic fields on health in various professional groups. The effect of sequential exposure to ionizing radiation and low-intensity broadband red light on the electrical activity of the heart and the microstructure of the rat myocardium was studied (Bavrina et al., 2015). Low intensity red light corrected some ECG parameters, in particular, normalized the QT and QTc intervals, as well as the voltage of the R and $\mathrm{T}$ waves. Changes in ECG parameters were accompanied by changes in the microstructure of myocardial muscle fibers in the experimental group compared to the control group.

A number of publications have examined the effects of electromagnetic radiation on specific gender groups. It has been established that the risk of glioma in adults increases with prolonged use (more than 10 years) of a mobile phone (Wang et al., 2018). The risks of breast cancer in men were assessed depending on the profession and the duration of exposure to extremely low frequency magnetic fields (Pollán et al., 2001). Various professional groups of men associated with magnetic fields are considered. A link between smartphone use and breast cancer risk among women (Shih et al., 2020) has been found. It has been noted that excessive smartphone use significantly increases the risk of breast cancer, especially for women with smartphone addiction. The negative effect occurs when the smartphone is located close to the chest, with the habit of using the smartphone before bed. An analysis of possible adverse consequences for the health of children and adolescents of exposure to electric, magnetic and electromagnetic fields was carried out (Otto \& Mühlendahl, 2007). It was found that there is no direct relationship between the effects of low-frequency magnetic fields on children and the risk of a brain tumour (Kheifets et al., 2010).

A number of publications have investigated the effect of a particular radiation source, in particular a mobile phone, on a person. The results of the work (Bortkiewicz et al., 2017) confirm the hypothesis that prolonged use of a mobile phone increases the risk of intracranial tumours, especially in the case of ipsilateral exposure. However, further research is needed to finally confirm this relationship. A significant positive association was found between prolonged use of a mobile phone (at least 10 years) and glioma (Yang et al., 2017; Wang et al., 2018). Other research (Magiera \& Solecka, 2019) emphasizes that the duration of human exposure to electromagnetic radiation will increase, and only further research can provide an answer about the possible negative effects of mobile phones and base stations. In the paper (Wall et al., 2019), various techniques for using a mobile phone are considered that reduce the negative impact on a person. Using a cell phone at a moderate distance from the head, using a speakerphone, using a wired headset or texting instead of talking, can reduce electromagnetic field exposure by up to two orders of magnitude in areas there is weak reception of signal. Using a mobile phone for web browsing, email, or streaming audio or video usually increases the distance between the phone and the body; however, the characteristics of electromagnetic interference associated with the transmission of data streams are different from the transmission of voice signals. In a paper Shih et al. (2020) found that the use of mobile phones increases the risk of breast cancer.

Separately, it is worth highlighting the publications in which the effect of electromagnetic radiation on the human heart is studied (Elmas, 2016).
This paper emphasizes that until the effect of the electromagnetic field on cardiac tissue is more fully understood electronic devices that generate an electromagnetic field should be used with caution. An article by Gumral et al. (2016) investigated the effect of an electromagnetic field with a frequency of $2450 \mathrm{MHz}$ on the heart and blood of rats. It was found that exposure of the heart to wireless $(2450 \mathrm{MHz})$ devices causes minor oxidative-antioxidant changes in the blood of rats, and moderate intake of melatonin may play an important role in the antioxidant system. One work Falcioni et al. (2018) investigated the effect of radiation from a GSM 1800 $\mathrm{MHz}$ base station on the brain and heart of rats. An increase in the incidence of brain and heart tumours was noted in rats after irradiation.

In a number of works, the influence of electromagnetic radiation on microscopic indicators of a biological object is considered. Work by Magro et al. (2005) and Drobakhin et al. (2007) studied the effect of millimeter-wave electromagnetic waves on some parameters of the immune system in clinically healthy people. The indicators of T-cell immunity, indicators of B-cell and humoral immunity were studied. The phagocytic reaction and bactericidal activity of peripheral blood have been studied. A test tube with human blood was exposed to microwave irradiation at frequencies of $42.2,50.3,51.7 \mathrm{GHz}$ with an exposure time of 15, 30, 60 minutes. Esmekaya et al. (2017) investigated the effect of electromagnetic radiation on mitochondria. The effect of microwave radiation with WCDMA modulation and $2.1 \mathrm{GHz}$ frequency on apoptotic activity and mitochondrial membrane potential in MCF-7 cells was studied. Cells were exposed to microwave radiation at a specific absorption rate (SAR) of $0.528 \mathrm{~W} / \mathrm{kg}$ for 4 or 24 hours.

At the same time, a number of international organizations, in particular IARC, are investigating the effect of electromagnetic fields on humans in the frequency range from $30 \mathrm{kHz}$ to $300 \mathrm{GHz}$. This study examines human exposure to radio frequency electromagnetic fields emitted by personal devices (mobile phones, cordless phones, Bluetooth, amateur radio transmitters), professional sources (high-frequency induction heaters, highpower pulse radars), environmental sources (mobile phone base stations, broadcasting antennas, medical devices). In this study, electromagnetic radiation is believed to act on a healthy person. However, there is no data on the effect of electromagnetic radiation on sick people in remission.

Among the diseases of the endocrine system, the most common are diseases of the thyroid gland. The absolute number of cases of thyroid disease in Ukraine over the past 10 years has increased from 689,000 to $1,846,000$. The prevalence rates of this disease increased, respectively, from 941.6 to 4,210.4 cases per 100 thousand of the population (Chukur, 2018). Thyroid pathology accounts for $46 \%$ of the total endocrinological morbidity, taking into account the fact that thyroid diseases include stage I-III goiter $(186.0 \%)$, nodular goiter $(9.8 \%)$, hypothyroidism $(6.4 \%)$, thyroiditis (5.9\%), thyrotoxicosis (1.7\%) and cancer of this gland (4.3\%). Such patients make extensive use of personal devices that radiate electromagnetic radiation in their daily lives. Therefore, it is very important to know what effect electromagnetic radiation has on such a group of patients.

Several publications have investigated the relationship between hyperthyroidism and heart disease. Thyroid hormones affect the cardiovascular system in different ways, which can greatly affect heart function. It has been established that hypothyroidism can lead to a decrease in cardiac output, an increase in systemic vascular resistance, a decrease in arterial elasticity and atherosclerosis (Udovcic et al., 2017). The assessment of cardiovascular morbidity and mortality in hyperthyroidism before and after treatment was carried out in the work by Ryödi et al. (2018) and Yang et al. (2019). Hyperthyroidism increases the risk of hospitalization for cardiovascular disease, and this risk persists for two decades after treatment with iodine-containing drugs or surgery. In their work (BieleckaDabrowa et al., 2019; Vale et al., 2019; Gencer et al., 2020), a long-term method of treating hypothyroidism is considered. The influence of herbal medicine on the treatment of hypothyroidism and cardiopulmonary dysfunction is discussed in (Chuang et al., 2020). A study of the offspring of pregnant rats with subclinical hypothyroidism and abnormal cardiac development was performed (Xue et al., 2020).

According to the treatment protocol, indications for removal of the thyroid gland (thyroidectomy procedure) are: malignant tumours, large compression goiter (multinodular or nodular), diffuse toxic goiter (Graves' disease, Graves goiter), follicular or adenomatous tumour neoplasia. Cur- 
rently, there is insufficient data on the effect of the thyroidectomy procedure on the morphogenesis of the heart. In modern conditions, such a group of patients can intensively use personal devices in everyday life. Therefore, it becomes necessary to investigate the complex effect of electromagnetic radiation on such a group of patients. Based on the above, it is of scientific interest to study the effect of electromagnetic radiation on a biological object under conditions of hypothyroidism. There is a need to study the mechanisms that shape human responses to thyroid disease. The purpose of our article was to assess the effect of electromagnetic radiation with different duration of exposure on the morphogenesis of the rat heart during thyroidectomy and in normal conditions.

\section{Materials and methods}

The Commission on Ethical Issues and Bioethics of the Dnepropetrovsk State Medical Academy (Protocol No. 1, January 16, 2017) established that the research program meets ethical requirements, according to the order of the Ministry of Health of Ukraine No. 231, 01.11.2005. When working with experimental animals, we adhered to the principles of the Declaration of Helsinki adopted by the General Assembly of the World Medical Association (1964-2000), the Council of Europe Convention on Human Rights and Biomedicine (1997), the provisions of the WHO, the International Council of Medical Scientific Societies, the International Code of Medical Ethics (1983), the law of Ukraine on "General ethical principles of experiments on animals" approved by the National Congress on Bioethics (2001), the provisions of the "European Convention for the Protection of Vertebrate Animals Used for Experimental and Other Scientific Purposes (1987)".

Laboratory rats were used as the object of research. The total number of rats intended for the experiment was 134. In the process of preparing rats for the experiment, namely, when modeling hypothyroidism, 8 animals or $6.0 \%$ died. The remaining 126 animals were divided into six experimental groups: control group; group after thyroidectomy; group for irradiation with an exposure duration of 45 minutes; group for irradiation with an exposure duration of 120 minutes; group after thyroidectomy and for irradiation with an exposure duration of 45 minutes; group after thyroidectomy and for irradiation with an exposure duration of 120 minutes.

Thus, there were 21 animals in each of the six groups. All rats in the groups were 6-month-old sexually mature males weighing 190-210 g. The rats were obtained from the vivarium of the Dnepropetrovsk Medical Academy. Before the experiment, all rats were examined and weighed. Their age, physical activity and skin condition were taken into account. During the entire period of preparation for the experiment and during the experiment, the rats were kept in the vivarium of the Medical Academy at a temperature of $20-25^{\circ} \mathrm{C}$, humidity not less than $50 \%$, in well-ventilated rooms, in standard plastic cages. Each cage contained no more than five rats.

A schematic of a microwave irradiation installation for rats is shown in Figure 1. Before starting the experiment, the power Pout at the generator output was measured. According to the passport data for the horn antenna, the effective area of the horn aperture Seff at the frequency $f=$ $10 \mathrm{GHz}$ is Seff $=245 \mathrm{~cm}^{2}$. For all series of the experiment, the condition Pout $/$ Seff $=1.110^{-3} \mathrm{~W} / \mathrm{cm}^{2}$ was satisfied. In principle, to enhance the effect of the electromagnetic field, the object could be placed in a resonator with holes (biconical resonator) to ensure breathing, but then the irradiation conditions would not correspond to those observed in practice (Andreev et al., 2014).

The rats were exposed to an electromagnetic field with a wavelength of $\lambda=3 \mathrm{~cm}$, which practically corresponds to the frequency range of satellite television. On the other hand, since the measurement of the maximum linear size of the rat gave an average of $\mathrm{L}=15 \mathrm{~cm}$, the ratio of the maximum linear size to the microwave wavelength was $L / \lambda=5$. When using this $L / \lambda$ ratio for a person, for the case of the maximum linear size of the human body $160-180 \mathrm{~cm}$, we obtain wavelengths $\lambda=32-36 \mathrm{~cm}$, which corresponds to frequencies of the order of $1 \mathrm{GHz}$. It is known that most wireless devices use frequencies of 1-3 GHz. It can be assumed that the fundamental processes of diffraction on the body of an animal and a person will be similar. Thus, on such an experimental model, it is possible to simulate the interaction of a person with an electromagnetic wave, the wavelength of which is close to the case of actually emitted waves for most communication systems. Moreover, in both cases, due to the ratio of sizes and wavelengths, the phenomenon of phased summation of reflected or transmitted waves can be excluded from consideration, which corresponds to the Bragg resonance (Borulko et al., 2014). This phenomenon has not found proper consideration in the analysis of the interaction of electromagnetic radiation with living objects.
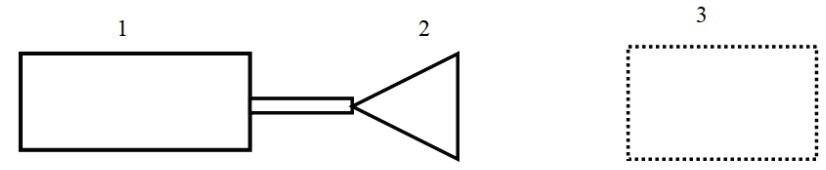

Fig. 1. Schematic of a laboratory setup for microwave irradiation of rats: electromagnetic oscillator (1); horn antenna (2); plastic container for placing animals (3)

It is obvious that direct observation of the consequences of thyroidectomy on the structure of the heart of a living person is impossible. Therefore, an experimental model has been developed for the analysis of morphogenetic changes in the heart during thyroidectomy. An additional external factor that influences morphological changes in the structure of the heart walls during thyroidectomy is microwave irradiation. Particular attention was paid to the choice of the duration of microwave irradiation. Two exposure durations were chosen, 45 and 120 minutes.

The hypothyroidism modeling technique was carried out as follows. Premedication was performed - atropine $0.25 \mathrm{mg} / \mathrm{kg}$ intramuscularly (IM), diphenhydramine $0.5 \mathrm{mg} / \mathrm{kg}$. The experimental animal was anesthetized with sodium thiopental, a non-inhalation anesthetic, $40 \mathrm{mg} / \mathrm{kg}$ intraperitoneally. The method for modeling hypothyroidism in rats includes access to the thyroid gland, its removal, thermocoagulation of the isthmus, cranial and caudal arteries of the thyroid gland and separation of the recurrent nerves according to utility model No. 54 (UA(11)27821(13)U); the parathyroid glands and the recurrent nerve are separated from the thyroid parenchyma. At the beginning, the front surface of the neck was shaved to the animals. The operation began with a longitudinal midline neck incision up to $3 \mathrm{~cm}$ long. The fascia was straightened around the neck and along the midline of the sternum - the hyoid muscles; holders were bluntly applied - two interrupted musculocutaneous sutures, along which the edges of the surgical wound were torn. $0.2 \mathrm{~mL}$ of $0.5 \%$ novocaine solution was injected into the thyroid capsule using an insulin syringe. The isthmus of the gland was burned with a thermocoagulator; the cranial and caudal vessels of both lobes of the gland were coagulated. Each lobe of the thyroid gland was separated from the corresponding part of the isthmus with two anatomical forceps in the caudocranial direction, separating the recurrent nerve and parathyroid glands from the thyroid parenchyma. At the same time, homeostasis was controlled, the dilated muscles were brought together with an octagonal suture, the surface of which was irrigated with a solution of ceftriaxone (Bicillin-5 according to model No. 54) to prevent purulent complications, and interrupted sutures were applied to the skin. Irradiation of animals lasted 10 days. On the 11th day, a myocardial examination was performed. The influence of the following factors on the dynamics of microscopic parameters in the myocardium of the rat heart was studied: procedures of thyroidectomy; the effect of general microwave irradiation of rats at different durations of exposure; the effect of general microwave irradiation of rats at different durations of exposure under hypothyroidism.

The study of the influence of electromagnetic radiation on the structure of the heart wall was carried out. To visualize changes in the vascular epithelium, the immunohistological marker CD-34 was used. With the help of it, the main stages of angiogenesis were determined. A comparison was made between the structure of the normal heart wall and the structure of the heart wall after microwave irradiation. The vascular endothelium was stained brown. The formation of the colour picture of the vascular endothelium was due to the accumulation of the CD-34 marker. Simultaneously with the monitoring of changes in the amount of the CD-34 marker in the heart wall areas, the placement of proliferative centers was monitored. A morphometric study of rat hearts was performed. A study of the effect of microwave radiation on the ultrastructural components of the rat myocardium was carried out. Morphometry was performed using a 
microscope Carl ZEISS, model PrimoStar. Image processing was performed using the program AxioVision LE Canon Module.

Statistical processing of the results of morphometry was carried out using the program Statistica 10.0 (StatSoft Inc., USA). We used the arithmetic mean (x) standard errors of the mean (SD) to describe the degree of general trend in quantitative traits.

\section{Results}

With a single exposure to only the first factor, destructive changes in the walls of the rat heart were observed in the myocardium under hypothyroidism after thyroidectomy (Fig. 2a). With the simultaneous exposure of rats to two factors, it can be seen that the visual results of such exposure, which were observed in the rat myocardium, differed significantly from the previous case (Fig. 2b, c). Figure 2b shows changes in the myocardium at an exposure duration of 45 minutes, Figure $2 c-$ at an exposure duration of 120 minutes. There was a significant difference in the structure of the myocardium at different exposure times. In Figure 3, the arrows show the destructive processes occurring in the myocardium under the influence of one (Fig. 3a) or two destructive external factors (Fig. 3b, c).

Figure 4 shows the vessels of the heart wall in normal conditions, after microwave irradiation with exposure duration of 45 minutes, after microwave irradiation with exposure duration of 120 minutes. The difference in the intensity of the colour of the vessels shows the degree of differentiation of the wall cells. The use of the CD-34 marker made it possible to establish that microwave irradiation with an exposure duration of $45 \mathrm{mi}-$ nutes not only increases the number of blood vessels, but also leads to differentiation of wall cells. The most significant increase in the relative volume of the endothelium in the areas of the rat heart myocardium was observed for the case of microwave irradiation only (without experimental hypothyroidism) with exposure duration of 45 minutes. In this case, the change in the relative volume of the endothelium was assessed by the accumulation of the CD-34 marker. At the same time, with exposure duration of 120 minutes, a decrease in this indicator in the heart wall was observed. Under conditions of exposure to two multiplicative factors (experimental hypothyroidism and microwave irradiation with exposure duration of 45 minutes), the accumulation of the vascular endothelial marker CD-34 was also established. But in this case, the accumulation was less pronounced than the accumulation of this marker without the conditions of experimental hypothyroidism (with exposure duration of $45 \mathrm{mi}-$ nutes). The following multidirectional trend is observed for the CD-34 marker: (1) both for the case of only microwave irradiation, and for the case of exposure to two multiplicative factors, there is an unambiguous tendency for the accumulation of the marker at an exposure of 45 minutes; (2) for the two above exposure cases, but with an exposure of $120 \mathrm{mi}-$ nutes, there is a tendency towards a decrease in the amount of marker.

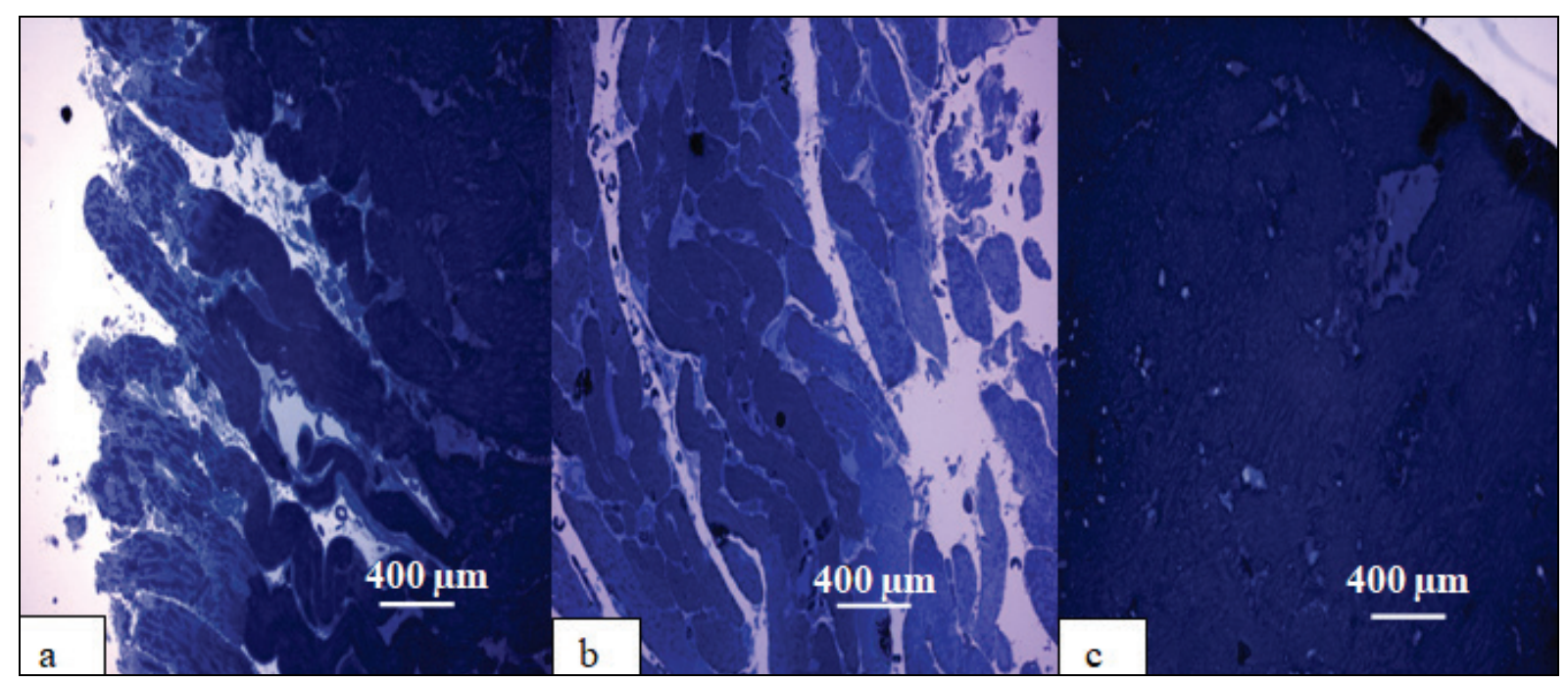

Fig. 2. Myocardium of the rat heart wall after thyroidectomy $(a)$, simultaneous exposure to thyroidectomy and microwave irradiation with exposure duration of 45 minutes $(b)$ and simultaneous exposure to thyroidectomy and microwave irradiation with exposure duration of 120 minutes $(c)$ : Mallory-Slinchenko stain, bar is $10 \mu \mathrm{m}$

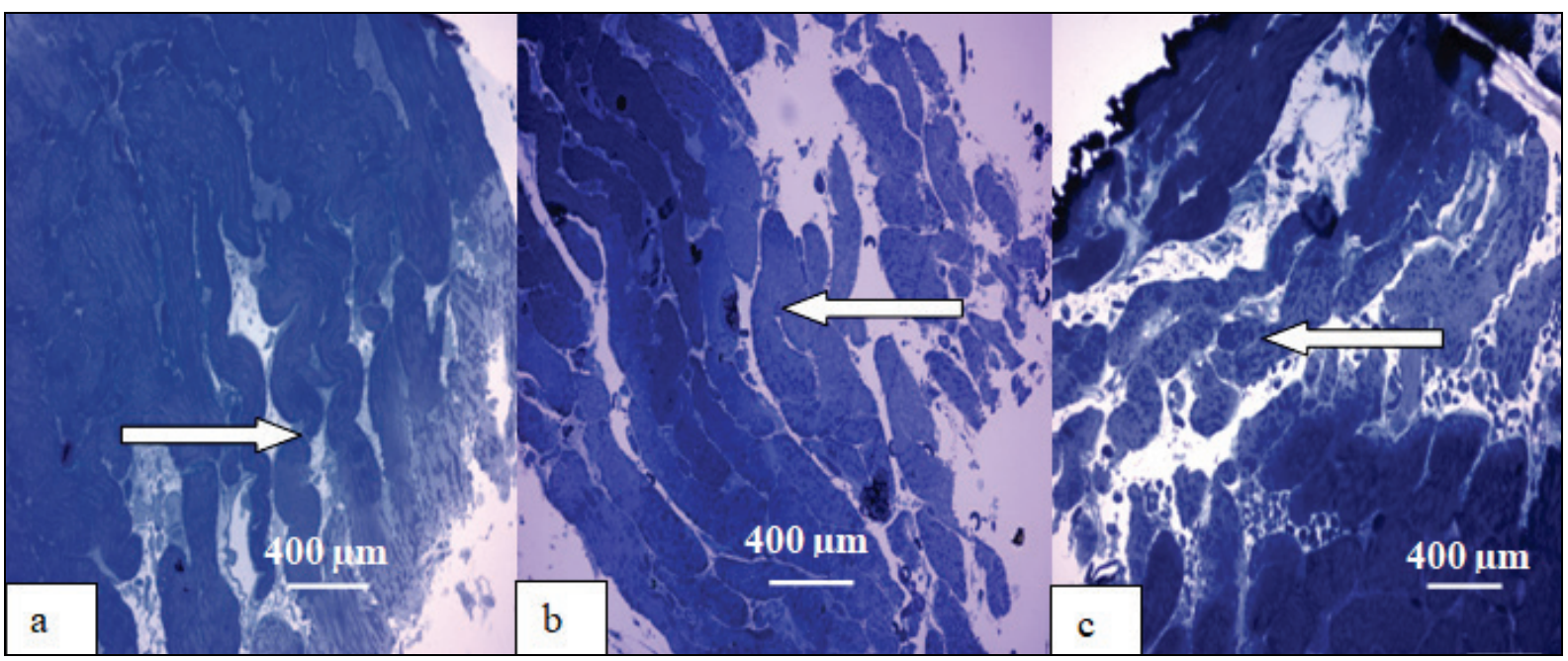

Fig. 3. Destructive processes in the myocardium after thyroidectomy $(a)$, microwave irradiation with exposure duration of 45 minutes in hypothyroidism $(b)$, microwave irradiation with an exposure time of 120 minutes under hypothyroidism $(c)$ : Mallory-Slinchenko stain, bar is $10 \mu \mathrm{m}$ 


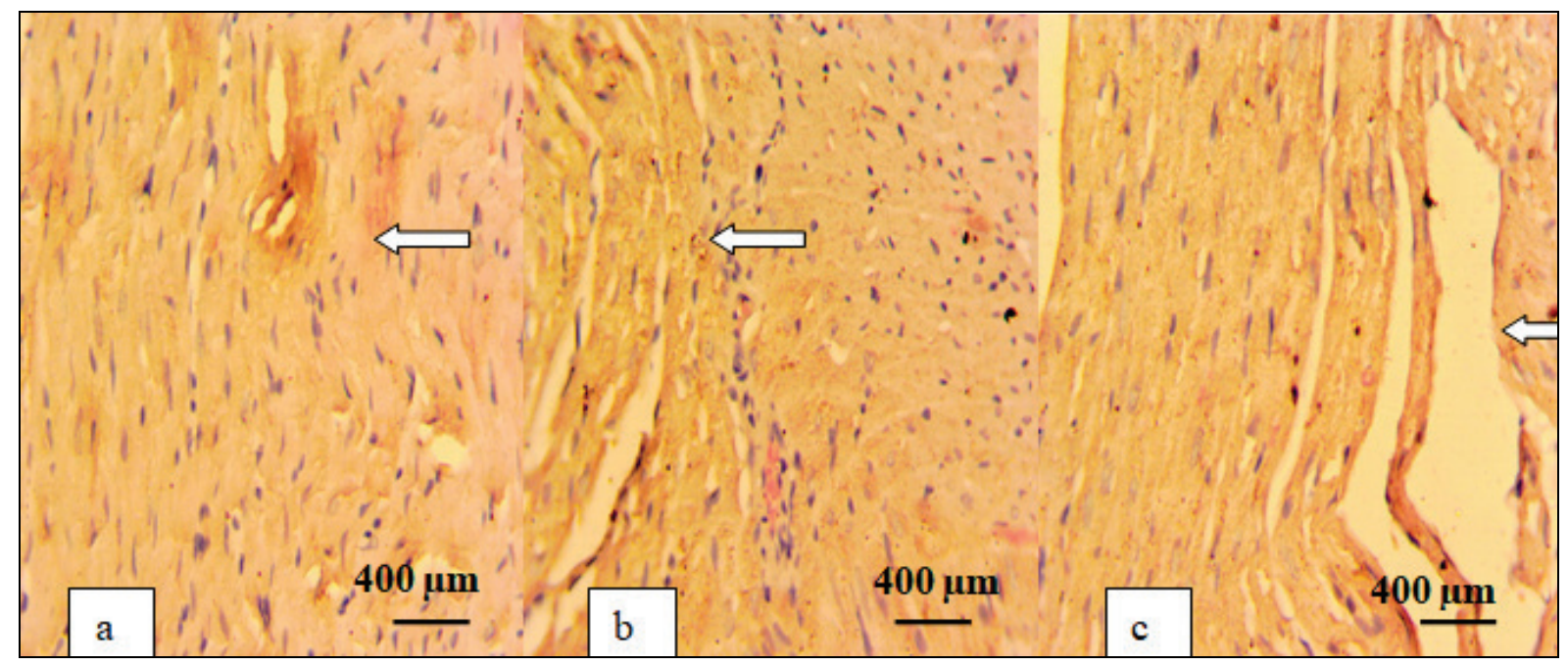

Fig. 4. Vessels of the heart wall in the absence of radiation (a), after microwave irradiation with exposure duration of 45 minutes $(b)$; after microwave irradiation with an exposure time of 120 minutes $(c)$, bar is $10 \mu \mathrm{m}$

Statistical processing of the results of morphometric study of rat hearts showed no changes in the wall thickness of the right ventricle of the heart (Table 1) and in the thickness of the wall of the left ventricle of the heart (Table 2) after microwave irradiation with an exposure duration of 45 and 120 minutes compared to the control group. It should be noted that there were practically no changes in the wall thickness of both the right and left ventricles after irradiation with exposure duration of 45 minutes. At the same time, after irradiation with duration of 120 minutes, there was a tendency for a scatter in the wall thickness values at different levels of the heart. In particular, this tendency is observed at the level of the lower third. Statistical processing of the results of a morphometric study of the cross-sectional area of the wall of the ventricles of the hearts of rats showed no changes in the cross-sectional area (Table 3 ). With exposure duration of 120 minutes, there is a tendency for a scatter in the values of this parameter in the right ventricle.

\section{Table 1}

Wall thickness of the right ventricle $(\mathrm{mm})$ of the heart at different levels of the cross-section in rats after microwave irradiation with different exposure times $(\mathrm{x} \pm \mathrm{SD}, \mathrm{n}=21)$

\begin{tabular}{cllcc}
\hline $\begin{array}{c}\text { Part } \\
\text { of heart }\end{array}$ & \multicolumn{1}{c}{$\begin{array}{c}\text { Levels } \\
\text { of cut heart }\end{array}$} & \multirow{2}{*}{ Control } & \multicolumn{2}{c}{ Microwave irradiation } \\
\cline { 4 - 5 } Front wall & basis & $0.73 \pm 0.05$ & $0.73 \pm 0.05$ & $0.76 \pm 0.03$ \\
& middle third & $0.93 \pm 0.04$ & $0.94 \pm 0.05$ & $0.96 \pm 0.05$ \\
& lower third & $0.91 \pm 0.05$ & $0.92 \pm 0.04$ & $0.94 \pm 0.05$ \\
& basis & $0.57 \pm 0.03$ & $0.57 \pm 0.05$ & $0.59 \pm 0.04$ \\
Back wall & middle third & $1.32 \pm 0.03$ & $1.32 \pm 0.04$ & $1.34 \pm 0.05$ \\
& lower third & $1.03 \pm 0.03$ & $1.04 \pm 0.04$ & $1.07 \pm 0.05$ \\
\hline
\end{tabular}

Note: statistical processing of the results of a morphometric study of rat hearts showed no changes in the wall thickness of the right ventricle of the rat heart.

Table 2

Left ventricular wall thickness $(\mathrm{mm})$ at three levels in rats

after microwave irradiation with different exposure times $(\mathrm{x} \pm \mathrm{SD}, \mathrm{n}=21)$

\begin{tabular}{clccc}
\hline $\begin{array}{c}\text { Part } \\
\text { of heart }\end{array}$ & $\begin{array}{c}\text { Levels } \\
\text { of cut heart }\end{array}$ & Control & \multicolumn{2}{c}{ Microwave irradiation } \\
Front wall & base & $1.94 \pm 0.07$ & $1.94 \pm 0.07$ & $1.96 \pm 0.08$ \\
& middle third & $2.82 \pm 0.05$ & $2.82 \pm 0.05$ & $2.84 \pm 0.07$ \\
& lower third & $1.22 \pm 0.04$ & $1.23 \pm 0.07$ & $1.24 \pm 0.07$ \\
& base & $1.89 \pm 0.05$ & $1.89 \pm 0.07$ & $1.91 \pm 0.07$ \\
Side wall & middle third & $2.33 \pm 0.05$ & $2.34 \pm 0.05$ & $2.35 \pm 0.70$ \\
& lower third & $1.35 \pm 0.02$ & $1.35 \pm 0.02$ & $1.37 \pm 0.04$ \\
& base & $2.27 \pm 0.06$ & $2.28 \pm 0.06$ & $2.29 \pm 0.09$ \\
Back wall & middle third & $2.82 \pm 0.12$ & $2.83 \pm 0.12$ & $2.84 \pm 0.12$ \\
& lower third & $1.92 \pm 0.03$ & $1.92 \pm 0.04$ & $1.94 \pm 0.05$ \\
\hline
\end{tabular}

Note: see Table 1.

After thyroidectomy, left ventricular cardiomyocytes in rat hearts contained numerous mitochondria, which were damaged in a vacuolarolytic manner. Paranuclear organelles are represented by small mitochondria with a light matrix and disordered cristae. Among the altered organelles, mitochondria were found with signs of uneven matrix clearing and destruction by cristae. Near the sarcolemma, the organelles had a spherical or elongated shape, a transparent matrix, with signs of edema and destruction of the cristae. There were few intermitochondrial contacts. The intermyofibrillar mitochondria were large. These mitochondria had moderately developed cristae and a transparent matrix (Fig. 5). Organelles with crystolysis and clearing of the mitochondrial matrix were found in a moderate amount. Also, sometimes giant mitochondria with a sharp edema of the matrix were found. The newly created micromitochondria were found in small numbers mainly near the nucleus.

Table 3

Cross-sectional area of the ventricular wall $\left(\mathrm{mm}^{2}\right)$ of rat hearts at three incision levels after microwave irradiation with different exposure times $(\mathrm{x} \pm \mathrm{SD}, \mathrm{n}=21)$

\begin{tabular}{clrrr}
\hline $\begin{array}{c}\text { Part } \\
\text { of heart }\end{array}$ & $\begin{array}{c}\text { Levels } \\
\text { of cut heart }\end{array}$ & Control & \multicolumn{2}{c}{ Microwave irradiation } \\
& base & $42.0 \pm 2.0$ & $42.0 \pm 2.3$ & $44.0 \pm 2.5$ \\
Right ventricle & middle third & $82.0 \pm 3.3$ & $83.0 \pm 3.4$ & $85.0 \pm 3.5$ \\
& lower third & $52.0 \pm 2.4$ & $53.0 \pm 2.5$ & $54.0 \pm 2.7$ \\
& base & $141.0 \pm 3.4$ & $142.0 \pm 3.3$ & $144.0 \pm 3.5$ \\
Left ventricle & middle third & $382.0 \pm 4.2$ & $383.0 \pm 4.3$ & $385.0 \pm 4.4$ \\
& lower third & $363.0 \pm 5.2$ & $364.0 \pm 5.3$ & $366.0 \pm 5.5$ \\
\hline
\end{tabular}

Note: see Table 1

After microwave irradiation of rats with exposure duration of $45 \mathrm{mi}-$ nutes, the study of the mitochondrial apparatus of the contractile cardiomyocytes of the left ventricle did not reveal significant differences between this experimental group and intact animals. The sub-sarcolemal zone of cardiomyocytes was characterized by the presence of polymorphic mitochondria. Among them were organelles of large volume, which had an elongated or spherical shape, an ordered orientation of the cristae, and a moderately dense matrix. Organelles were found smaller in volume, smaller in the development of outer and inner mitochondrial membranes and they had a small number of weakly oriented cristae. Also, small mitochondria were found, the number and, accordingly, the density of cristae in which significantly exceeded those of other types of mitochondria. The paranuclear zone of contractile myocytes was represented mainly by mitochondria with ultrastructural signs of moderate functional activity. One can observe small globular organelles, with a clear matrix and a small amount of low density, weakly oriented cristae. Many neighboring mitochondria were in contact with each other through intermitochondrial contacts. Sometimes the outer mitochondrial membrane was completely absent in the area of junction of two organelles, and in this place an intermitochondrial bridge was formed, which connected the matrix of both mitochondria. Such bridges were located at the level of the Z-lines of myofibrils. Studies of mitochondria that were localized near the nucleus 
showed that irregularly organized organelles formed more contacts than the orderly located mitochondria between the myofibrillar zones (Fig. 6).

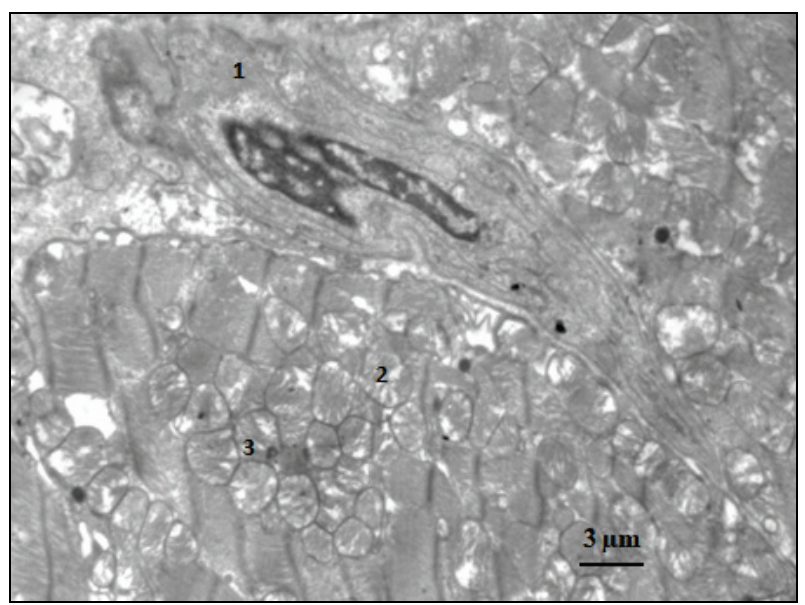

Fig. 5. Ultrastructure of the mitochondrial apparatus of the contractile cardiomyocytes of the left ventricle of the rat heart after thyroidectomy: capillary (1), cardiomyocyte (2), mitochondria (3)

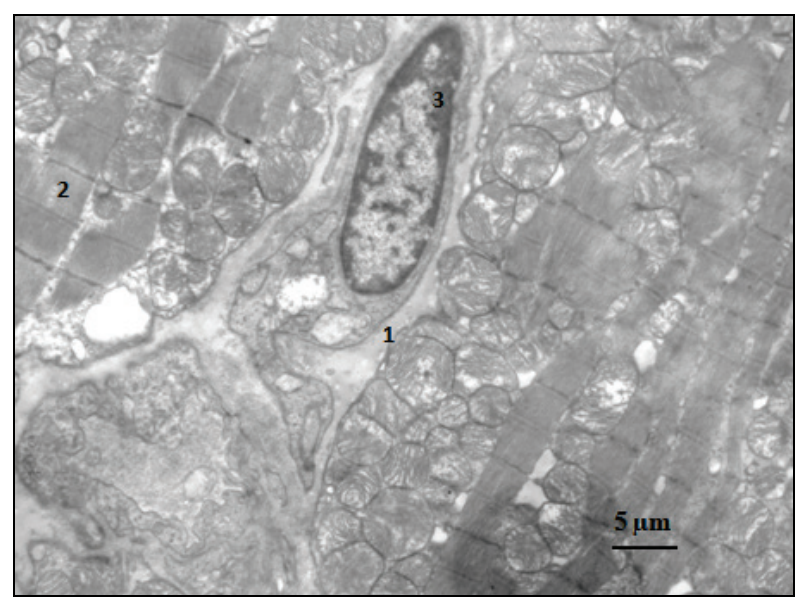

Fig. 6. Ultrastructure of the mitochondrial apparatus of the contractile cardiomyocytes of the left ventricle of the rat heart after electromagnetic irradiation with an exposure of 45 minutes: capillary $(1)$, cardiomyocyte (2), endotheliocyte (3)

After electromagnetic irradiation of rats with the exposure of $120 \mathrm{mi}-$ nutes, a study of the state of subsarcolemal organelles showed that the processes of degradation in this zone of cells were significantly enhanced and prevailed over the processes of reproduction. There were a large number of mitochondria that burst, empty and shriveled organelles, in the hyaloplasm, finely dispersed amorphous masses. Mitochondria practically did not form associations with each other. The overwhelming majority of mitochondria had cracks and ruptures of the outer membrane, matrix outflow, destruction of cristae. The density of intermitochondrial contacts in this group was sharply reduced in comparison with the indicators of control animals. Near the nucleus, mitochondria were mainly in a state of overstrain, they were freely located in the hyaloplasm around the nucleus or in places formed clusters of organelles and actively formed contacts with each other, although the density of intermitochondrial contacts is lower than in intact animals. The mitochondria between the myofibrils were heteromorphic, but they were mostly in a state of pronounced edema. Degradation of organelles was mainly carried out by rupture of the outer membrane and leakage of the matrix into the hyaloplasm. The number of destroyed mitochondria was small. Signs of the disintegration of associations of mitochondria, which weakly formed contacts with each other, were established. After microwave irradiation with an exposure of 45 minutes, in the contractile cardiomyocytes of the left ventricle of the heart of animals in a hypothyroid state, mitochondria in paranuclear localization were represented by enlarged organelles with a vacuolar type of damage. Organellamas showed no signs of destruction of the outer membranes; they showed moderate crystallysis, an electron-light matrix. Some mitochondria had relatively stable small cristae with a moderately dense matrix (Fig. 7). A large number of micromitochondria with structurally stable outer membranes and cristae and a homogeneous matrix without electron-dense inclusions were observed. A large number of small intermitochondrial contacts were found. In the subsarcolemal zone, the organelles were spherical or elongated. Organelles had a moderately electron-dense matrix. Mitochondria, organelles with destroyed cristae and areas of matrix clearing were visible. There is no damage in the structure of the outer mitochondrial membrane. In the spaces between myofibrils, mitochondria with signs of structural and functional overstrain predominated. Along with this, numerous newly created micromitochondria were observed in large numbers.

Left ventricular cardiomyocytes in the heart of rats with thyroidectomy after exposure to electromagnetic irradiation with an exposure of $120 \mathrm{mi}-$ nutes contained numerous organelles which were damaged by the vacuolar-lytic type. Organelles of paranuclear localization were represented by small mitochondria with a light matrix and disordered cristae. Among the altered organelles, mitochondria with signs of overvoltage were encountered: areas of uneven clarification of the matrix, with phenomena of destruction of cristae. There was a small number of intermitochondrial contacts between these mitochondria (Fig. 8). In the subsarcolemal zone, the organelles had a spherical or elongated shape, a transparent matrix. Significantly swollen mitochondria, organelles with destroyed cristae and areas of sharp matrix clearing were observed. Cracks and ruptures were observed in the outer mitochondrial membrane. There are few intermitochondrial contacts (Fig. 9).

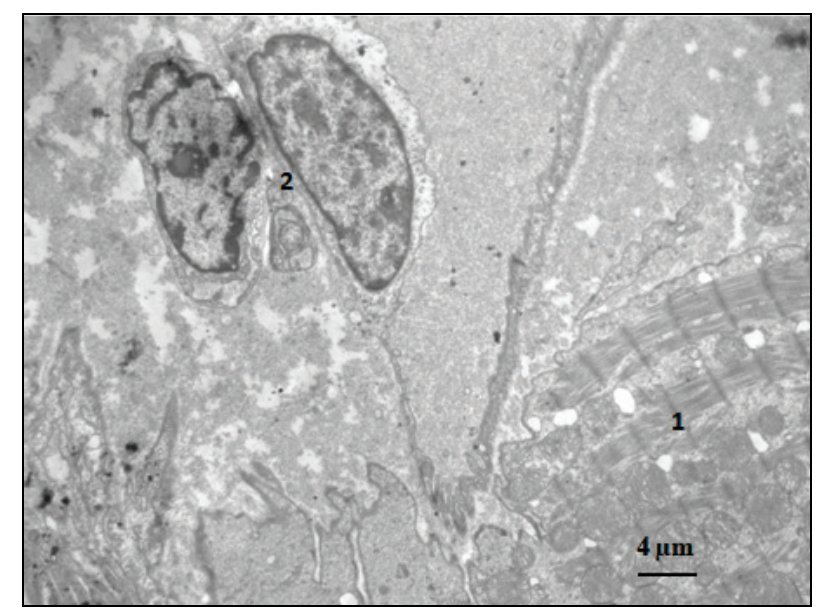

Fig. 7. Ultrastructure of the mitochondrial apparatus of the contractile cardiomyocytes of the left ventricle of the rat heart after electromagnetic irradiation with an exposure of 120 minutes: cardiomyocyte (1), endotheliocytes (2)

\section{Discussion}

In the course of research, a case of simultaneous exposure of rats to two multiplicative factors: thyroidectomy and microwave irradiation with exposure duration of 45 minutes was considered. It was found that for this case, there is a compensatory restructuring of the constant structural components of the myocardium of the heart wall and hypertrophy of cardiomyocytes. This fact shows that under microwave irradiation with exposure duration of 45 minutes, a reparative-regenerative effect on the myocardium is observed. For the case of the simultaneous effect of the two factors considered above, but with exposure duration of 120 minutes, an increase in degenerative and destructive processes in the myocardium of the heart wall is observed (Hardell, 2017). This process can be explained by the depressing effect of microwave irradiation on cardiomyocytes. In this case, there are microinfarctions with perifocal inflammation, as well as degeneration processes, which are characterized by the appearance of contracture strips of muscle fibers and the appearance of disseminated necrosis of cardiomyocytes (Gumral et al., 2016).

The modeling and remodeling of hypothyroidism after thyroidectomy was carried out. For these cases, a study of the structure of the myo- 
cardium was performed. The general tendencies in the myocardium have been established: destructive changes are observed; there is a process of degeneration of muscle fibers (Bielecka-Dabrowa et al., 2019; Yang et al., 2019).

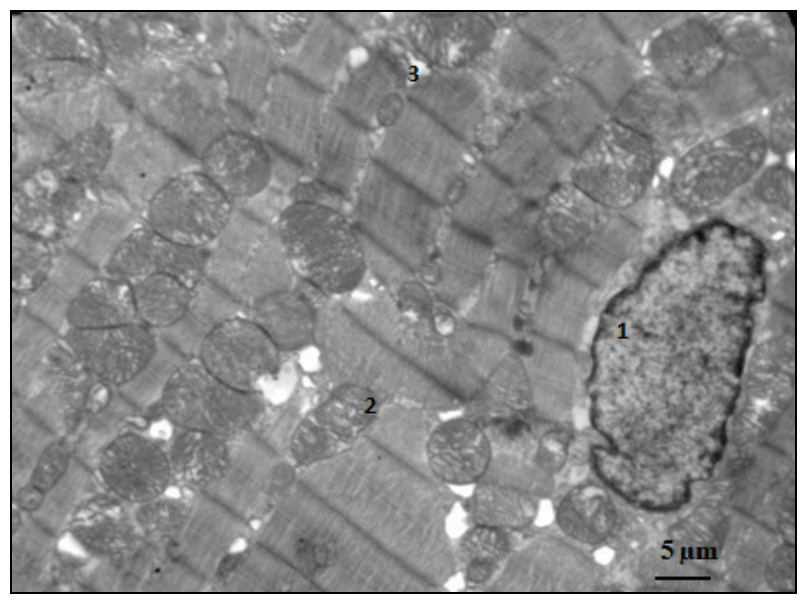

Fig. 8. Ultrastructure of paranuclear and intermyofibrillar mitochondria of contractile cardiomyocytes of the left ventricle of a rat heart after electromagnetic irradiation with an exposure of 45 minutes under hypothyroidism: endotheliocyte (1), mitochondria (2), cardiomyocyte (3)

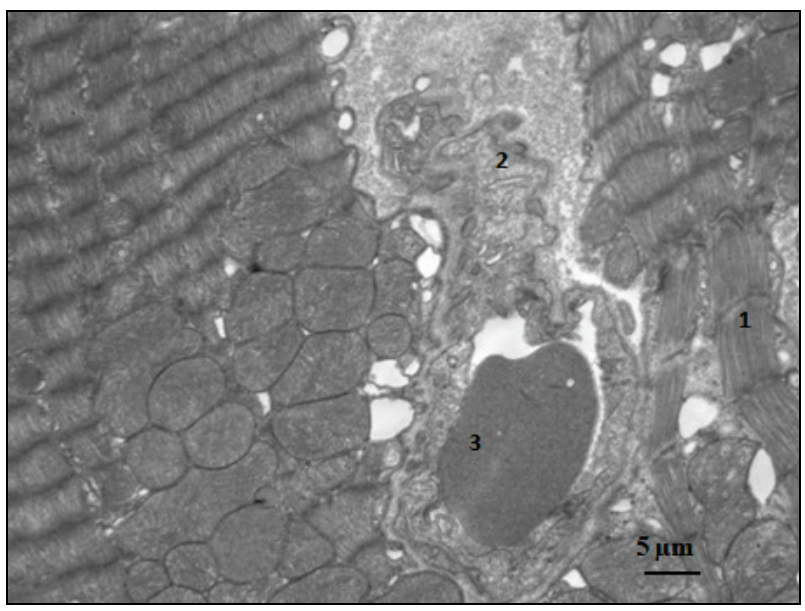

Fig. 9. Ultrastructure of the mitochondrial apparatus of the contractile cardiomyocytes of the left ventricle of the rat heart after electromagnetic irradiation with an exposure of 120 minutes under hypothyroidism: cardiomyocyte (1), capillary (2), erythrocyte sludge syndrome (3)

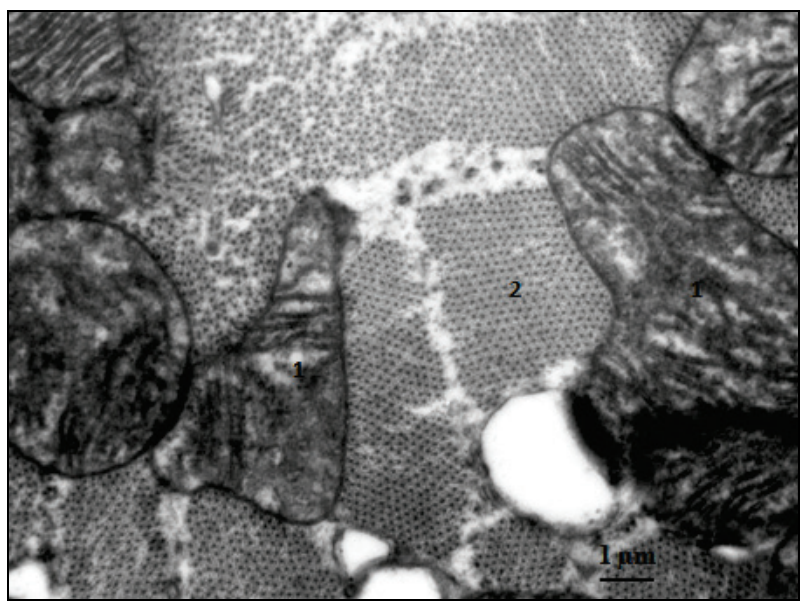

Fig. 10. Ultrastructure of intermyofibrillar mitochondria of contractile cardiomyocytes of the left ventricle of a rat heart after electromagnetic irradiation with an exposure of 120 minutes under hypothyroidism: mitochondria (1), myofilaments (2)
It should be noted that after microwave irradiation with the exposure duration of 45 minutes, a regenerative compensatory restructuring of the structural components of the myocardium of the heart wall occurs. On the other hand, with simultaneous exposure to thyroidectomy and microwave irradiation with exposure duration of 120 minutes, an increase in degenerative and destructive processes in the myocardium of the heart wall is observed. This indicates the depressing effect of prolonged microwave irradiation on cardiomyocytes (Udovcic et al., 2017; Vale et al., 2019). Thus, we can draw the following conclusion: the duration of microwave irradiation is a decisive factor that determines the final result of the multiplicative effect on the myocardium of two factors. At short exposure times of microwave irradiation (up to 45 minutes), a stimulating effect on the myocardium is observed, at long exposures (120 minutes or more) - a depressive one. A similar trend is also true for the effect of microwave irradiation on blood (Magro et al., 2005; Kosharniy et al., 2017; Drobakhin et al., 2020).

Other objects of research in the course of the experiment were: the processes of vasodilation and the process of formation of new blood vessels (Bavrina et al., 2015). It was established that under the influence of the above factors, changes occur in the walls of the heart, especially the myocardium. Vascular expansion and angiogenesis processes are observed, which significantly depend on the duration of microwave irradiation. At the same time, there is a violation of blood circulation in the myocardium of the heart of rats due to changes in the thickness of the walls of the heart. The use of the immunohistochemical marker CD-34, which is a marker of vascular endothelium, made it possible to determine the main stages of angiogenesis. Uneven accumulation of the marker was observed in different parts of the heart walls, both in normal conditions and after exposure to only one factor - microwave irradiation with exposure duration of 45 and 120 minutes. A similar trend was also observed under the influence of the two multiplicative factors discussed above (Ryödi et al., 2018). As it might be expected, normally the greatest amount of the CD34 marker was observed, of course, in the walls of the heart vessels. The greatest amount of the CD-34 marker in the structure of blood vessels in the rat heart wall was observed after exposure to one factor - microwave irradiation with exposure duration of 45 minutes. At the same time, when exposed to a single factor - microwave irradiation with exposure duration of 120 minutes, significantly less accumulation of CD-34 was observed in the structure of blood vessels in the rat heart wall. With the multiplicative effect of experimental hypothyroidism and microwave irradiation on rats, the greatest amount of the vascular endothelial marker CD34 was observed in the heart wall after exposure to microwave irradiation with exposure duration of 45 minutes. This fact shows the activity of angiogenesis in areas of the heart. This process is a consequence of compensatory activity under conditions of experimental hypothyroidism. On the other hand, with exposure duration of 120 minutes, a minimal amount of marker was observed in the heart wall. And such a process can be considered as decompensatory activity under conditions of experimental hypothyroidism. The latter fact is confirmed by immunohistochemical studies.

The intermyofibrillar mitochondria were large. These mitochondria had moderately developed cristae and a transparent matrix. Among such organelles, organelles with elements of cristolysis and zones of sharp matrix clearing prevailed (Fig. 10). Deepening of damage to the structure of intermitochondrial contacts was noted. The main part of mitochondria was represented by organelles with irreversible or reversible damage, as well as overvoltage phenomena. Sometimes there were giant mitochondria due to a sharp edema of the matrix. Newly created micromitochondria were found in small numbers against the background of destructive and degenerative processes. These mitochondria were observed as small spherical formations with single cristae. Cristae were localized mainly near the nucleus.

\section{Conclusion}

Uneven accumulation of the CD-34 marker showed that the highest degree of differentiation of vascular cells in the myocardium of the rat heart is observed after exposure to microwave irradiation with exposure duration of 45 minutes. It was found that after exposure to microwave irradiation with exposure duration of 45 minutes under hypothyroidism, 
the number of blood vessels increases. However, the amount of this marker in the rat heart myocardium decreased after an exposure of 120 minutes both for the case of microwave irradiation only and for the case of microwave irradiation under conditions of experimental hypothyroidism.

Thus, the results of the studies carried out made it possible to determine the general patterns of changes in the heart wall after exposure to various factors considered and at different durations of exposure. The dynamics of the main histogenetic processes in the rat under normal conditions and under experimental conditions was established. It has been shown that experimental hypothyroidism in rats manifests itself in the form of increased heteromorphism of mitochondria of contractile cardiomyocytes of the left ventricle, cristolysis of intermyofibrillar mitochondria, the appearance of giant organelles with a sharp edema of the matrix. After electromagnetic irradiation with the exposure of 45 minutes, the general structure of the mitochondrial apparatus does not change. Irradiation with the exposure duration of 120 minutes causes the destruction of subsarcolemal paranuclear organelles, edema and degradation of intermyofibrillar mitochondria. After exposure to electromagnetic radiation with an exposure of 45 minutes in a hypothyroid state, a compensatory restructuring of the energy apparatus of the contractile cardiomyocytes of the left ventricle occurs due to the formation of mitochondria and an increase in their contact interaction. After exposure to radiation for 120 minutes, the development of destructive-degenerative processes in the mitochondrial apparatus of the left ventricular cardiomyocytes, deepening of damage to the intermitochondrial contacts is observed. More research is needed to investigate the effect of a modulated high-frequency electromagnetic field on rats. It is necessary to establish the basic regularities for other durations of exposure $\mathrm{T}$ ( $\mathrm{min}$.), in particular, in the time interval $45<\mathrm{T}<120$ and $\mathrm{T}<45$.

This work was supported by the Ministry of Education and Science of Ukraine (0119U101221).

\section{References}

Andreev, M. V., Drobakhin, O. O., Privalov, Y. N., \& Saltykov, D. Y. (2014). Measurement of dielectric material properties using coupled biconical resonators. Telecommunications and Radio Engineering, 73(11), 1017-1032.

Bavrina, A. P., Monich, V. A., Malinovskaya, S. L., Yakovleva, E. I., Bugrova, M. L., \& Lazukin, V. F. (2015). Method for correction of consequences of radiation-induced heart disease using low-intensity electromagnetic emission under experimental conditions. Bulletin of Experimental Biology and Medicine, 159(1), 103-106.

Belyaev, A., Dean, H., Eger, G., Hubmann, G., Jandrisovits, R., Kern, M., Kundi, M., Moshammer, H., Lercher, P., Müller, K., Oberfeld, G., Ohnsorge, P., Pelzmann, P., Scheingraber, C., \& Thill, R. (2016). EUROPAEM EMF Guideline 2016 for the prevention, diagnosis and treatment of EMF-related health problems and illnesses. Reviews on Environmental Health, 31(3), 363-397.

Bielecka-Dabrowa, A., Godoy, B., Suzuki, T., Banach, M., \& von Haehling, S. (2019). Subclinical hypothyroidism and the development of heart failure: An overview of risk and effects on cardiac function. Clinical Research in Cardiology, 108(3), 225-233.

Bortkiewicz, A., Gadzicka, E., \& Szymczak, W. (2017). Mobile phone use and risk for intracranial tumors and salivary gland tumors - A meta-analysis. International Journal of Occupational Medicine and Environmental Health, 30, $27-43$.

Borulko, V. F., Drobakhin, O. O., \& Sidorov, D. V. (2014). Pulse shape transformation under Bragg structures irradiation. Telecommunications and Radio Engineering, 73(2), 95-109.

Chuang, T. Y., Lien, C. Y., Hsu, C. H., Lu, C. W., \& Wu, C. H. (2020). Chinese herbal medicine alleviates thyroidectomy-induced cardiopulmonary exercise dysfunction in rats. Evidence-Based Complementary and Alternative Medicine, 2, 1-8.

Chukur, O. A. (2018). Dynamika zakhvoryuvanosti y poshyrennya patolohiyi shchytopodibnoyi zalozy sered dorosloho naselennya Ukrayiny [Dynamics of morbidity and spread of thyroid pathology among the adult population of Ukraine]. Visnyk Sotsial'noyi Hihiyeny ta Orhanizatsiyi Okhorony Zdorov'ya Ukrayiny, 4(78), 19-25 (in Ukrainian).

Drobakhin, O. O., Likholat, Y. V., Magro, V. I., Ryabchiy, V. D., Kudrjavtseva, V.E., Tatarchuk, O. M., \& Pokataev, V. N. (2007). Influence of electromagnettic radiation on the seeds of technical plants and the blood of man. In: Kostenko, A. A., \& Nosich, A. I. (Eds.). Kharkov symposium on physics and engineering of microwaves, millimeters and sumillimeter. Kharkiv, Ukraine. Pp. 879-881.

Drobakhin, O., Magro, V., Kosharnyi, V., Rutgaizer, V., \& Abdul-Ohly, L. (2020). Microscopic changes in the myocardium of the heart wall of rats under the influence of microwave electromagnetic radiation under the conditions of mode- ling hypothyroidism. In: Antyufeyeva, M. S. (Ed.). Ukrainian Microwave Week. Kharkiv, Ukraine. Pp. 586-589.

Elmas, O. (2016). Effects of electromagnetic field exposure on the heart: A systematic review. Toxicology and Industrial Health, 32(1), 76-82.

Esmailzadeh, S., Delavar, M. A., Aleyassin, A., Gholamian, S. A., \& Ahmadi, A. (2019). Exposure to electromagnetic fields of high voltage overhead power lines and female infertility. International Journal of Occupational and Environmental Health, 10(1), 11-16.

Esmekaya, M. A., Canseven, A. G., Kayhan, H., Tuysuz, M. Z., Sirav, B., \& Seyhan, N. (2017). Mitochondrial hyperpolarization and cytochrome-c release in microwaveexposed MCF-7 cells. General Physiology and Biophysics, 36(2), 211-218.

Falcioni, L., Bua, L., Tibaldi, E., Lauriola, M., De Angelis, L., Gnudi, F., Mandrioli, D., Manservigi, M., Manservisi, F., Manzoli, I., Menghetti, I., Montella, R., Panzacchi, S., Sgargi, D., Strollo, V., Vornoli, A., \& Belpoggi, F. (2018). Report of final results regarding brain and heart tumors in Sprague-Dawley rats exposed from prenatal life until natural death to mobile phone radiofrequency field representative of a $1.8 \mathrm{GHz}$ GSM base station environmental emission. Environmental Research, 165, 496-503.

Gencer, B., Moutzouri, E., Blum, M. R., Feller, M., Collet, T. H., Delgiovane, C., da Costa, B. R., Buffle, E., Monney, P., Gabus, V., Müller, H., Sykiotis, G. P., Kearney, P., Gussekloo, J., Westendorp, R., Stott, D. J., Bauer, D. C., \& Rodondi, N. (2020). The impact of levothyroxine on cardiac function in older adults with mild subclinical hypothyroidism: A randomized clinical trial. The American Journal of Medicine, 133(7), 848-856.

Gumral, N., Saygin, M., Asci, H., Uguz, A. C., Celik, O., Doguc, D. K., Savas, H. B., \& Comlekci, S. (2016). The effects of electromagnetic radiation $(2450 \mathrm{MHz}$ wireless devices) on the heart and blood tissue: Role of melatonin. Bratislavske Lekarske Listy, 117(11), 665-671.

Hardell, L. (2017). World Health Organization, radiofrequency radiation and healtha hard nut to crack (review). International Journal of Oncology, 51(2), 405-413.

Jalilian, H., Eeftens, M., Ziaei, M., \& Röösli, M. (2019). Public exposure to radiofrequency electromagnetic fields in everyday microenvironments: an updated systematic review for Europe. Environmental Research, 176, 108517.

Kheifets, L., Ahlbom, A., Crespi, C., Feychting, M., Johansen, C., Monroe, J., Murphy, M. F. G., Oksuzyan, S., Preston-Martin, S., Roman, T., Saito, E., Savitz, D., Schüz, J., Simpson, J., Swanson, J., Tynes, T., Verkasalo, P., \& Mezei, G. (2010). A pooled analysis of extremely low-frequency magnetic fields and childhood brain tumors. American Journal of Epidemiology, 172(7), 752-761.

Kim, J., Seo, S., Lee, D. N., Park, S., Im, K. J., Park, S., \& Jin, Y. W. (2020). Occupational exposure characteristics and factors associated with radiation doses among Korean radiation workers. Radiation Protection Dosimetry, 189(2), 106-113.

Kosharnyi, V. V., Rutgaizer, V. H., Abdul-Ohly, L. V., Magro, V. I., \& Kumchenko, V. V. (2017). Zmina pokaznykiv krovi u shchuriv pislya diyi elektromahnitnoho vyprominyuvannya [Change in blood parameters in rats after exposure to electromagnetic radiation]. Klinichna ta Eksperymentalna Patolohiya, 60, 28 32 (in Ukrainian).

Magiera, A., \& Solecka, J. (2019). Mobile telephony and its effects on human health. Roczniki Państwowego Zakładu Higieny, 70(3), 225-234.

Magro, V. I., Drobakhin, O. O., Alekseev, V. V., Kudrjavtseva, V. E., \& Tatarchuk, O. M. (2005). Influencing of electromagnetic radiation on some immunological indexes of organism. In: Shifrin, Y. S., \& Dubrovka, F. F. (Eds.), International Conference on Antenna Theory and Techniques. IEEE, Kyiv. Pp. 489-491.

Melnick, R. L. (2019). Commentary on the utility of the national toxicology program study on cell phone radiofrequency radiation data for assessing human health risks despite unfounded criticisms aimed at minimizing the findings of adverse health effects. Environmental Research, 168, 1-6.

Meroni, D., \& Schreck, S. (2015). Electromagnetic fields: Activities in the European Commission with a focus on research projects and the Scientific Committee of Emerging and Newly Identified Health Risks (SCENIHR). Electromagnetic Biology and Medicine, 34(3), 171-174.

Miah, T., \& Kamat, D. (2017). Current understanding of the health effects of electromagnetic fields. Pediatric Annals, 46(4), 172-174.

Otto, M., \& Mühlendahl, K. E. (2007). Electromagnetic fields (EMF): Do they play a role in children's environmental health $(\mathrm{CEH})$. International Journal of Hygiene and Environmental Health, 210(5), 635-644.

Pollán, M., Gustavsson, P., \& Floderus, B. (2001). Breast cancer, occupation, and exposure to electromagnetic fields among Swedish men. American Journal of Industrial Medicine, 39, 276-285.

Ryödi, E., Metso, S., Huhtala, H., Välimäki, M., Auvinen, A., \& Jaatinen, P. (2018). Cardiovascular morbidity and mortality after treatment of hyperthyroidism with either radioactive iodine or thyroidectomy. Thyroid, 28(9), 1111-1120.

Sagar, S., Dongus, S., Schoeni, A., Roser, K., Eeftens, M., Struchen, B., Foerster, M., Meier, N., Adem, S., \& Röösli, M. (2017). Radiofrequency electromagnetic field exposure in everyday microenvironments in Europe: A systematic literature review. Journal of Exposure Science and Environmental Epidemiology, 28, 147-160.

Shih, Y.-W., Hung, C.-S., Huang, C.-C., Chou, K.-R., Niu, S.-F., Chan, S., \& Tsai, H.-T. (2020). The association between smartphone use and breast cancer risk 
among Taiwanese women: A case-control study. Cancer Management and Research, 12, 10799-10807.

Shih, Y.-W., O’brien, A. P., Hung, C.-S., Chen, K.-H., Hou, W.-H., \& Tsai, H.-T. (2020). Exposure to radiofrequency radiation increases the risk of breast cancer: A systematic review and meta-analysis. Experimental and Therapeutic Medicine, 21(1), 1-3.

Udovcic, M., Pena, R. H., Patham, B., Tabatabai, L., \& Kansara, A. (2017). Hypothyroidism and the heart. Houston Methodist DeBakey Cardiovascular Joumal, 13(2), 55-59.

Vale, C., Neves, J. S., von Hafe, M., Borges-Canha, M., \& Leite-Moreira, A. (2019). The role of thyroid hormones in heart failure. Cardiovascular Drugs and Therapy, 33(2), 179-188.
Wall, S., Wang, Z. M., Kendig, T., Dobraca, D., \& Lipsett, M. (2019). Real-world cell phone radiofrequency electromagnetic field exposures. Environmental Research, 171, 581-592.

Wang, P., Hou, C., Li, Y., \& Zhou, D. (2018). Wireless phone use and risk of adult glioma: Evidence from a meta-analysis. World Neurosurgy, 115, 629-636.

Xue, D., Sun, J. L., \& Yang, J. (2020). Early L-T4 intervention improves fetal heart development in pregnant rats with subclinical hypothyroidism rats by activating BMP4/Smad4 signaling pathway. BMC Cardiovascular Disorders, 20(1), 369.

Yang, G., Wang, Y., Ma, A., \& Wang, T. (2019). Subclinical thyroid dysfunction is associated with adverse prognosis in heart failure patients with reduced ejection fraction. BMC Cardiovascular Disorders, 19(1), 83.

Yang, M., Guo, W., Yang, C., Tang, J., Huang, Q., Feng, S., Jiang, A., Xu, X., \& Jiang, G. (2017). Mobile phone use and glioma risk: A systematic review and meta-analysis. PLoS One, 12(5), e0175136. 